\title{
Hepatic Epithelioid Hemangioendothelioma - a Rare Tumor and Diagnostic Dilemma
}

\author{
VLADISLAV TRESKA ${ }^{1}$, ONDREJ DAUM ${ }^{2}$, MARTIN SVAJDLER ${ }^{2}$, \\ VACLAV LISKA $^{1}$, JIRI FERDA ${ }^{3}$ and JAN BAXA ${ }^{3}$ \\ ${ }^{1}$ Department of Surgery, Faculty of Medicine and Faculty Hospital Pilsen, \\ Charles University Prague, Pilsen, Czech Republic; \\ ${ }^{2}$ Sikl's Institute of Pathology, Faculty of Medicine and Faculty Hospital Pilsen, \\ Charles University Prague, Pilsen, Czech Republic; \\ ${ }^{3}$ Department of Radiodiagnostics, Faculty of Medicine and Faculty Hospital Pilsen, \\ Charles University Prague, Pilsen, Czech Republic
}

\begin{abstract}
Background/Aim: Hepatic epithelioid haemangioendothelioma (HEHE) is a very rare malignant tumor of vascular origin and uncertain biological behaviour that is difficult to diagnose using preoperative radiology diagnostic techniques. Patients and Methods: The authors present here two patients with HEHE of different extent. The first patient had a generalised form of disease, with involvement of the liver, lungs and bones and was treated with a combination of bevacizumab and capecitabine. The second patient had a localised form of disease involving the liver and this was resolved using a combination of liver resection and radiofrequency ablation. In both patients, the radiology work-up before surgery was non-specific and metastases of another malignant process were considered. The definitive histological diagnosis was made by the pathologist on the basis of immunohistochemical analysis that demonstrated the presence of CD31, CD34 and calmodulin-binding transcription activator 1 (CAMTA 1). Results: Both patients remain in an overall good condition 27 and 5 months respectively following treatment for HEHE. Conclusion: Preoperative radiological diagnosis of HEHE is difficult and immunohistochemical examination of the tumor tissue
\end{abstract}

This article is freely accessible online.

Correspondence to: Prof. Vladislav Treska, Department of Surgery, Faculty of Medicine and Faculty Hospital Pilsen, Charles University Prague, Alej Svobody 80, 30460 Pilsen, Czech Republic. Tel: +420 3771042701, +420 602444700, Fax: +420 377103965, e-mail: treska@fnplzen.cz

Key Words: Hepatic epithelioid haemangioendothelioma, diagnostic dilemma, treatment options. sample remains the key diagnostic tool. Radical surgical resection or liver transplantation is the method of choice in patients with localised liver involvement.

Hepatic epithelioid haemangioendothelioma (HEHE) represents a very rare vascular tumor of the liver of epithelioid origin. The biological behaviour of this malignant tumor is uncertain and usually falls between that of benign haemangioma and that of malignant angiosarcoma. Basic diagnosis using all available methods is very difficult and this entity is often mistaken for other primary or secondary liver tumors. The definitive diagnosis is determined only by the histopathologist. We illustrate here the difficulties of the primary diagnostic workup of HEHE and treatment options on two patients.

\section{Patients and Methods}

The first case involved a 50-year old patient who underwent computed tomography (CT, Definition Flash, Siemens, Germany) for dyspnoea in January 2015, which demonstrated a metastatic focus in the left lung. The positron emission tomography (PET CT, Biograph mCT 128, Siemens, Germany) revealed further foci in the liver and an osteolytic focus in the vertebral column (Figure 1). A liver biopsy under CT guidance was performed. HEHE was diagnosed according to positivity of CD31, CD34 and negativity of the cytokeratins OSCAR (Sigma-Aldrich Co. LLC, USA), AE 1/3 (BioLegend, Switzerland) and CAM 5.2 (Sigma-Aldrich Co. LLC, USA). Given that the process was generalised, the multidisciplinary team decided oncological treatment with bevacizumab (ROCHE, Switzerland) and capecitabine (Glenmark Pharmaceuticals, Czech Republic). Patient signed the Informed Consent.

The second case involved a 75-year old patient who on a routine ultrasound examination (USG, Acuson S 2000, Siemens, Germany) in February 2016 was found to have a focal process of the liver of unknown origin. The PET CT indicated the possibility of a tumor of the caecum with metastasis to the liver involving segments 2, 3, 


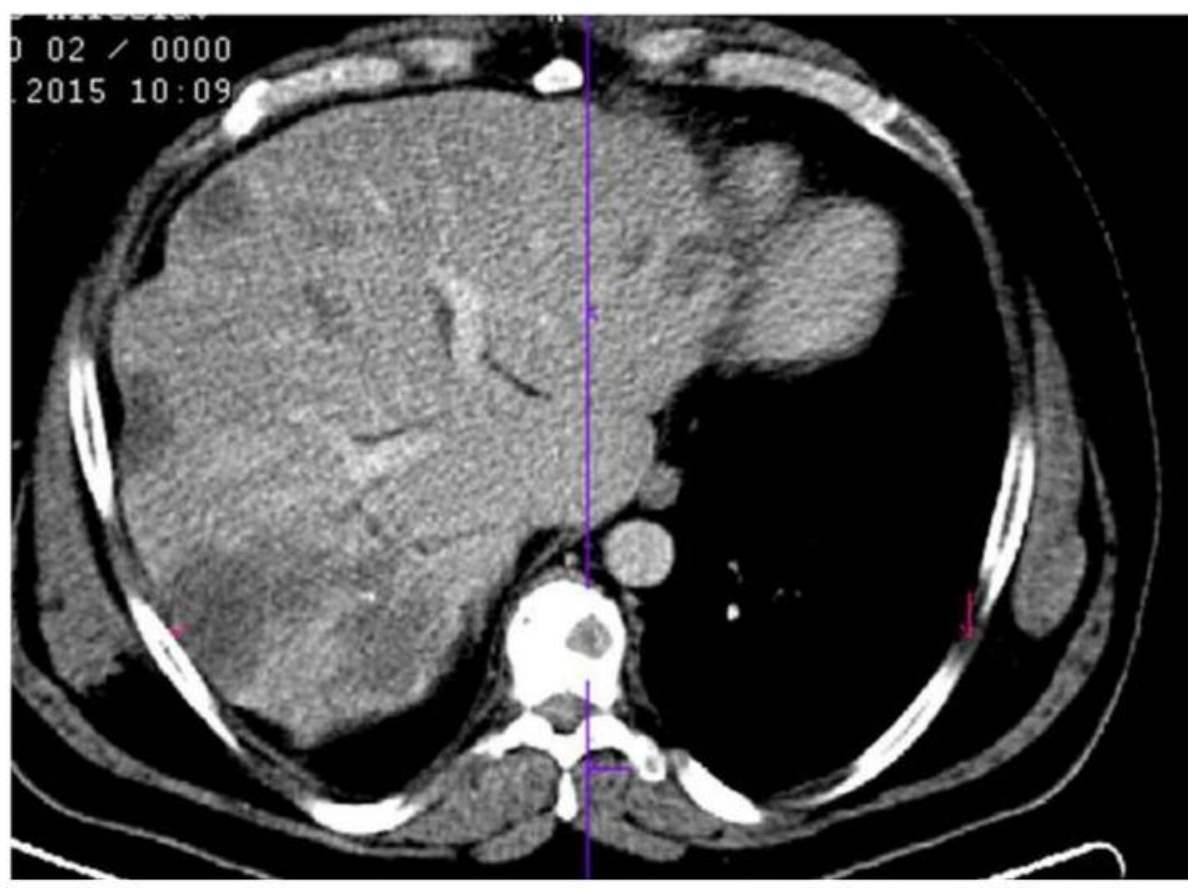

Figure 1. Multiple liver metastases and osteolytic focus in the vertebral column - PET CT (portal venous phase, Biograph mCT 128, Siemens, Germany).

4 and 5 with a diameter of up to $3 \mathrm{~cm}$ (Figure 2). Colonoscopy uncovered the presence of an adenoma of the caecum. Pre-operative serum tumor markers demonstrated a mild elevation of Cancer antigen 72-4 (CA 72-4, ROCHE, Switzerland) - (12.1 kIU/1 - norm 0-7 kIU/l) and Tissue polypeptide specific antigen (TPS, Beki Diagnostics AB, Sweden) $121 \mathrm{IU} / 1$ (norm 0-90 IU/l) levels. All other tumor markers were within normal range. We performed a right hemicolectomy. Preoperative USG demonstrated suspected liver metastasis. Nonetheless, the postoperative histological examination of colon confirmed the presence of an adenoma with low-grade dysplasia. The postoperative course was uncomplicated. The patient underwent magnetic resonance imaging (MRI, Magnetom Skyra, Siemens, Germany) of the liver, which confirmed the presence of multiple liver foci that appeared to be metastases and metastases of a neuroendocrine tumor could not be ruled out either. Liver biopsy under CT guidance was performed. HEHE was diagnosed on the immunohistochemical stain using the calmodulinbinding transcription activator 1 (CAMTA 1) antibody (Novus Biologicals, Littleton, CO, USA) (Figures 3 and 4). We performed a trisegmentectomy with radiofrequency ablation of the focus in the 5 th liver segment. The postoperative course was uncomplicated. Given the radicality of the surgical procedure, adjuvant oncologica treatment was not indicated. Patient signed the Informed Consent.

\section{Results}

The first patient is now two years after treatment with stable disease according to the Response Evaluation Criteria in Solid Tumors (RECIST). The second patient is now one year after the procedure in good condition.

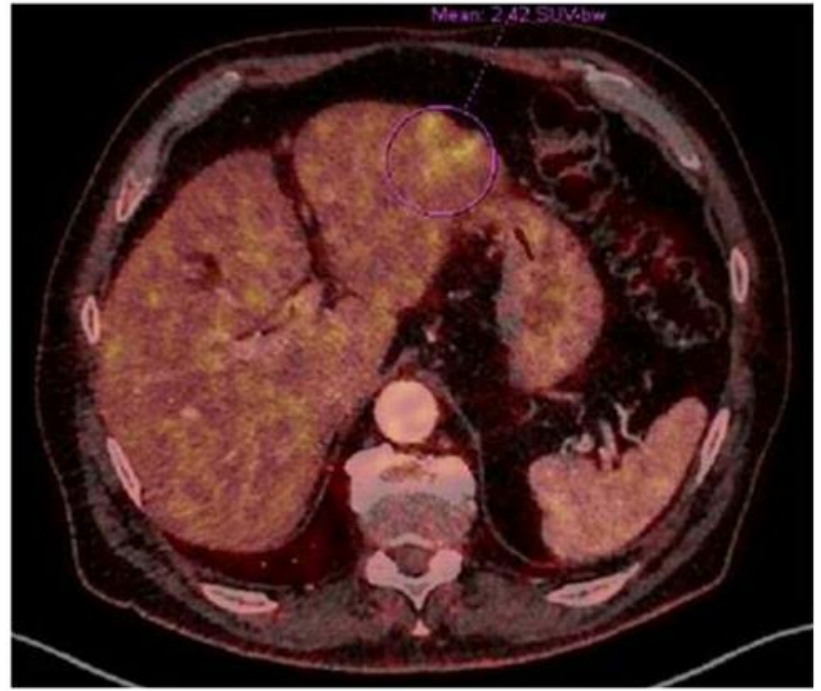

Figure 2. Metastasis of the segment 3 (alpha range PET CT, Biograph mCT 128, Siemens, Germany).

\section{Discussion}

HEHE is a tumor originating from the endothelial cells of uncertain malignant potential. The incidence of HEHE is estimated to be $1 / 1$ million inhabitants. It most frequently affects patients between the ages of 30 to 40 years. The 


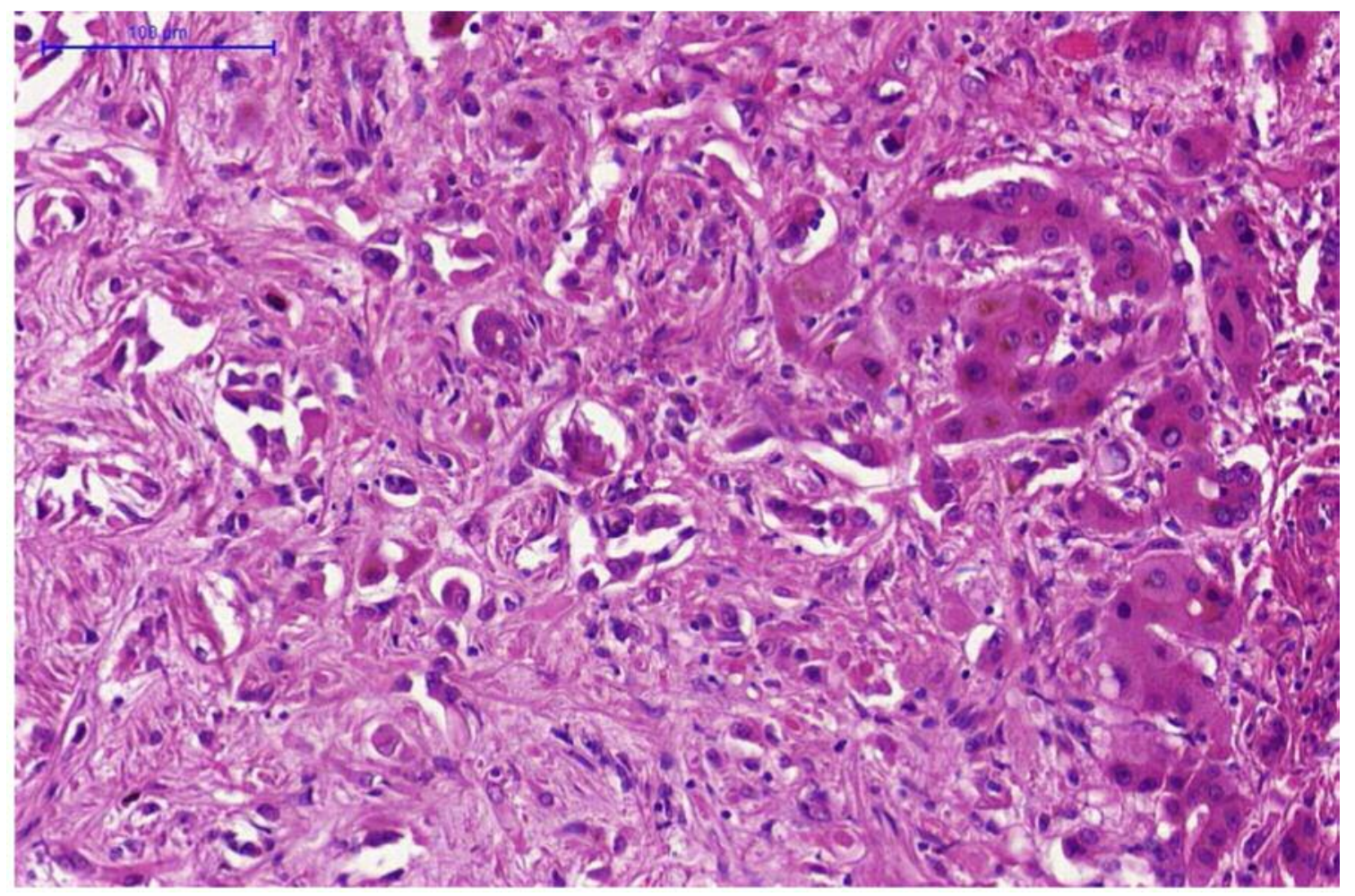

Figure 3. Left to the liver parenchyma there is a proliferation of neoplastic cells with voluminous cytoplasm forming discrete lumens apparent in abundant fibrous stroma $(H \& E, 200 \times)$.

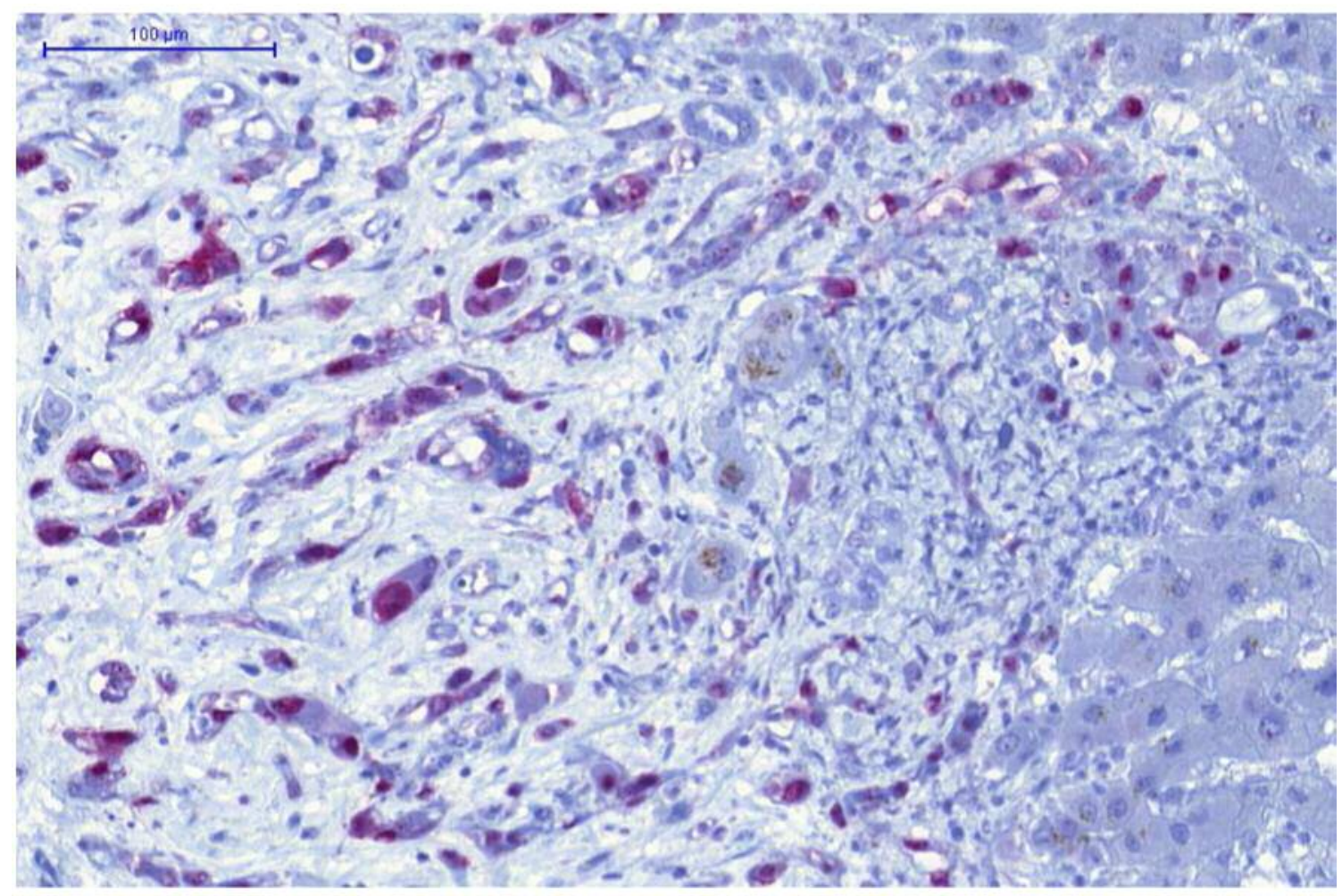

Figure 4. Neoplastic cells readily stain for calmodulin binding transcription activator 1 (CAMTA1 antibody, 200x). 
cause of its development remains unknown. Risk factors are thought to include contraceptives, trauma to the liver, prior hepatitis, long-term contact with asbestos, vinyl chloride or thorotrast. However, no clear causal connection between the development of HEHE and the cited risk factors has been demonstrated to date $(1,2)$.

HEHE presents with varied symptoms. It may manifest as abdominal discomfort, pain in the region of the liver, BuddChiari or Kasabach-Merritt syndrome. Rupture of the tumor may occur in the case of large HEHE with intraabdominal bleeding. In around $50 \%$ of patients, the haemangioendothelioma may occur in other locations, especially other visceral organs, lymph nodes, bones and lungs (3).

HEHE is often diagnosed incidentally using USG, CT, MRI, PET CT, or positron emission magnetic resonance tomography (PET MRI), indicated for other reasons. Contrast-enhanced USG, CT or MRI are important for the diagnosis of HEHE. Typical findings of haemangioendothelioma include multiple foci located at the periphery of the liver parenchyma with retraction of the liver surface. The signal emitted by the foci on MRI as well as the appearance on CT imaging is very similar to other hyper-vascularised tumors or their metastases. A typical sign of HEHE is the concentric enhancement of the vascularised component by the contrast agent, whereby the centre corresponding to fibrotic tissues practically fails to be enhanced. Fine calcifications may also appear in some tumors. In more advanced cases, the foci coalesce and gradually replace the liver parenchyma. However, given the tumor's relatively slow growth, the unattained liver parenchyma often hypertrophies significantly. Nonetheless, many findings are non-specific and are diagnosed as liver metastases or primary liver tumors $(4,5)$. In our two cases, the findings were not typical of HEHE, and liver metastases were considered to be the underlying diagnosis. This was also supported in the second patient by the prior ileocecal resection of an adenoma of the caecum.

The therapeutic method of choice involves radical resection or, in bilobar forms, liver transplantation. Both methods demonstrate good $70 \%$ five-year overall survival (6, $7)$. In cases where surgical treatment cannot be performed, oncological treatment is indicated (8). No specific agent or drug combination has been discovered. Given the expression of vascular endothelial growth factor (VEGF) in HEHE, targeted treatment of HEHE is based on anti-VEGF agents, namely bevacizumab either in monotherapy or in combination with cytostatic drugs, most often capecitabine (9). This combination was also successfully used in our first patient. Given the biological behaviour of the disease, a conservative, watchful-waiting approach is possible also in long-term stable forms of HEHE.

The prognosis of patients with HEHE is controversial. Patients with multiple nodules have a worse prognosis. In contrast, the prognosis is not affected by the presence of extra-hepatic metastases (10). Worse overall survival has been noted in patients with HEHE over $10 \mathrm{~cm}$ in diameter and in older patients. The average one-year survival following various treatment modalities ranges from 88-100\% and five-year survival ranges from $23-83 \%$ depending on the biological behaviour of the tumor (11). Nonetheless, surgical treatment yields the best long-term results.

In most patients, the definitive diagnosis is made by the histopathologist. The characteristic histopathological features of HEHE include histochemical positivity of CD 34 and CD 31. Nuclear CAMTA1 expression, which is verified in $85-90 \%$ of patients, is currently the main diagnosis of HEHE. The other method with high sensitivity and specificity is fluorescence in situ hybridization or reverse transcription polymerase chain reaction to detect the WW domaincontaining transcription regulator (WWTRI)-CAMTA1 fusion gene. However, this method is not routinely available $(12,13)$.

HEHE is a very rare tumor of uncertain biological behaviour with non-specific symptoms. It is difficult to diagnose and its prognosis is often uncertain. Treatment choices include radical surgical resection or liver transplantation.

\section{Acknowledgements}

This study was supported by the Research project of Charles University, Prague - PROGRES.

\section{References}

1 Ishak KG, Sesterhenn IA, Goodman ZD, Rabin L and Stromeyer FW: Epitheloid hemangioendothelioma of the liver: a clinicopathologic and follow-up study of 32 cases. Hum Pathol 15: 839-852, 1984.

2 Hu HJ, Jin YW, Jing QY, Shrestha A, Cheng NS and Li FY: Hepatic epithelioid hemangioendothelioma: Dilemma and challenges in the preoperative diagnosis. World J Gastroenterol 22: 9247-9250, 2014.

3 Mistry AM, Gorden DL, Busler JF, Coogan AC, Kelly BS: Diagnostic and therapeutic challenges in hepatic epithelioid hemangioendothelioma. J Gastrointest Cancer 43: 521-525, 2012.

4 Thin LW, Wong DD, De Boer BW, Ferguson JM, Adams L and Macquillan G: Hepatic epithelioid haemangioendothelioma: challenges in diagnosis and management. Intern Med J 40: 710$715,2010$.

5 Mehrabi A, Hoffmann K, Weiss KH, Mogler C, Schemmer P and Büchler MW: Long term follow up after resection emphasizes the role of surgery in Primary Hepatic Epithelioid Hemangioendothelioma. Ann Med Surg (Lond) 10: 1-4, 2016.

6 Remiszewski P, Szczerba E, Kalinowski P, Gierej B, Dudek K and Grodzicki M: Epithelioid hemangioendothelioma of the liver as a rare indication for liver transplantation. World $\mathrm{J}$ Gastroenterol 20: 11333-11339, 2016.

7 Oz I, Lacin S, Kucuk O and Bilgic S: Selective intra-arterial Y90 microsphere therapy in hemangioendothelioma. Turk J Gastroenterol 22: 89-92, 2011. 
8 Thomas RM, Aloia TA, Truty MJ, Tseng WH, Choi EA and Curley SA: Treatment sequencing strategy for hepatic epithelioid haemangioendothelioma. HPB (Oxford) 16: 677-685, 2014.

9 Lau A, Malangone S, Green M, Badari A, Clarke K and Elquza E: Combination capecitabine and bevacizumab in the treatment of metastatic hepatic epithelioid hemangioendothelioma. Ther Adv Med Oncol 7: 229-236, 2015.

10 Grotz TE, Nagorney D, Donohue J, Que F, Kendrick M and Farnell M: Hepatic epithelioid haemangioendothelioma: is transplantation the only treatment option? HPB (Oxford) 12: 546-553, 2010.
11 Doyle LA, Fletcher ChDM and Hornick JL: Nuclear Expression of CAMTA1 Distinguishes Epithelioid Hemangioendothelioma From Histologic Mimics. Am J Surg Pathol 40: 94-102, 2016.

12 Shibuya R, Matsuyama A, Shiba E, Harada H, Yabuki K and Hisaoka M: CAMTA1 is a useful immunohistochemical marker for diagnosing epithelioid haemangioendothelioma. Histopathology 67: 827- 835, 2015.

Received May 20, 2017

Revised May 29, 2017

Accepted May 31, 2017 